\title{
First Genomic Analysis of Dendritic Cells from Lung and Draining Lymph Nodes in Murine Asthma
}

\author{
Thomas Tschernig, ${ }^{1}$ Christina Hartwig, ${ }^{2}$ Andreas Jeron, ${ }^{3}$ Quoc Thai Dinh, \\ Marcus Gereke, ${ }^{3,5}$ and Dunja Bruder ${ }^{3,5}$ \\ ${ }^{1}$ Institute of Anatomy and Cell Biology, Saarland University, Campus Homburg, Kirrberger Straße 100, 66424 Homburg, Germany \\ ${ }^{2}$ Institute of Pharmacology, Medical School of Hannover, Carl-Neuberg-Straße 1, 30625 Hannover, Germany \\ ${ }^{3}$ Infection Immunology Group, Institute of Medical Microbiology, Infection Control and Prevention, \\ Otto-von-Guericke University Magdeburg, Leipziger Straße 44, 39120 Magdeburg, Germany \\ ${ }^{4}$ Department of Experimental Pneumology, Saarland University, Campus Homburg, Kirrberger Straße 100, 66424 Homburg, Germany \\ ${ }^{5}$ Immune Regulation Group, Helmholtz Centre for Infection Research, Inhoffenstraße 7, 38124 Braunschweig, Germany
}

Correspondence should be addressed to Dunja Bruder; dunja.bruder@med.ovgu.de

Received 16 November 2014; Revised 9 February 2015; Accepted 9 February 2015

Academic Editor: Giuliana Napolitano

Copyright (C) 2015 Thomas Tschernig et al. This is an open access article distributed under the Creative Commons Attribution License, which permits unrestricted use, distribution, and reproduction in any medium, provided the original work is properly cited.

\begin{abstract}
Asthma is the consequence of allergic inflammation in the lung compartments and lung-draining lymph nodes. Dendritic cells initiate and promote $\mathrm{T}$ cell response and drive it to immunity or allergy. However, their modes of action during asthma are poorly understood. In this study, an allergic inflammation with ovalbumin was induced in 38 mice versus 42 control animals. After ovalbumin aerosol challenge, conventional dendritic cells (CD11c/MHCII/CD8) were isolated from the lungs and the draining lymph nodes by means of magnetic cell sorting followed by fluorescence-activated cell sorting. A comparative transcriptional analysis was performed using gene arrays. In general, many transcripts are up- and downregulated in the CD8 $8^{-}$dendritic cells of the allergic inflamed lung tissue, whereas few genes are regulated in $\mathrm{CD}^{+}$dendritic cells. The dendritic cells of the lymph nodes also showed minor transcriptional changes. The data support the relevance of the $\mathrm{CD} 8^{-}$conventional dendritic cells but do not exclude distinct functions of the small population of $\mathrm{CD} 8^{+}$dendritic cells, such as cross presentation of external antigen. So far, this is the first approach performing gene arrays in dendritic cells obtained from lung tissue and lung-draining lymph nodes of asthmatic-like mice.
\end{abstract}

\section{Introduction}

Dendritic cells play a key role not only in asthma during the initiation of the allergic immune response but also in the effector phase of the allergic inflammation leading to typical clinical symptoms [1,2]. Allergy pathophysiology hereby reveals both similarities and clear differences between humans and mice. Basically, the dendritic cells can be divided into three groups: a small population of plasmacytoid dendritic cells, a predominant population of conventional dendritic cells, and, during inflammation, the monocytederived or inflammatory dendritic cells [3]. The dendritic cells isolated and analysed in this study were the so-called conventional dendritic cells, which are positive for CD1lc and MHCII [4]. In addition, the expression of CD8 was used to separate $\mathrm{CD} 8^{+}$from $\mathrm{CD} 8^{-}$dendritic cells. Upon comparison, fewer $\mathrm{CD}^{+}$dendritic cells than $\mathrm{CD} 8^{-}$ones were found in the lung tissue. The $\mathrm{CD} 8^{+}$dendritic cells were more concentrated in the draining lymph nodes, making them a lymph noderesident dendritic cell population $[4,5]$. Furthermore, within lymph nodes the $\mathrm{CD} 8^{+}$dendritic cells contribute to cytotoxic $\mathrm{T}$ cell responses via cross presentation of exogenous antigens $[2,4,6]$. $\mathrm{CD}^{-}$, but not the $\mathrm{CD}^{+}$, sorted dendritic cells from schistosoma-infected mice prevented allergic responses [7]. $\mathrm{CD}^{+}$and $\mathrm{CD} 8^{-}$dendritic cells from BCG-infected mice suppressed allergic $\mathrm{T}$ cell responses in vitro and in vivo [8].

In recent years, the expression of CD103 and CD1lb has been introduced for phenotyping dendritic cells in asthma and elsewhere. The lymphoid resident dendritic cells are characterized as $\mathrm{CD} 103^{-}$dendritic cells $\left(\mathrm{CD} 1 \mathrm{~b}^{+}, \mathrm{CD}^{+}\right.$, and 
$\mathrm{CD}^{-}$). In contrast the nonlymphoid residents are characterized as $\mathrm{CD} 103^{+}$dendritic cells $\left(\mathrm{CD} 1 \mathrm{~b}^{+}, \mathrm{CD}^{+}\right.$, and $\mathrm{CD} 8^{-}$) [3]. Our approach to the gene expression of conventional dendritic cells compared $\mathrm{CD}^{-}$and $\mathrm{CD}^{+}$conventional dendritic cells, revealing an interesting panel of regulated genes. Since there is a close relation between dendritic cells in the tissue and the draining lymph nodes, both compartments were taken for analysis. The majority of dendritic cells pick up allergen not only in the bronchi but also in the alveoli and migrate to lymph nodes where the allergen is presented to $\mathrm{B}$ cell and $\mathrm{T}$ cells initiating and maintaining humoral and cellular lymphocyte responses. Lymphocytes become activated and recirculate through the tissues including the lung where dendritic cell immigration and activation are mediated $[1,2]$.

\section{Aims}

The present study had the aim to compare the gene expression of distinct dendritic cells isolated from the lung tissue and the lung-draining lymph nodes in mice with induced asthmaticlike inflammation and controls. A further aim of the presented study was to compare lung tissue and lymph nodederived dendritic cells from control animals and animals suffering from allergic inflammation. Obtaining enough cell numbers of dendritic cell subsets for gene expression analysis is challenging. The more the subsets that are defined using multiple markers, the more the difficult the harvesting of a sufficient number of dendritic cells. Therefore, a strategy was chosen to obtain sufficient numbers of dendritic cells in a medium scale approach, using less than fifty animals each for the disease group and the control group. The classical combination of CD11c and MHCII defined the small numbers of conventional dendritic cells which yielded the draining mediastinal lymph nodes. For the bigger lung tissue yield of dendritic cells, the expression of CD $8 \alpha$, which is relevant for pulmonary allergy, was additionally included. Data from gene arrays in murine macrophages and dendritic cells from lung tissue is available [9], but to our knowledge there is no data on gene arrays in dendritic cells from allergic or asthmatic-like inflamed lungs. Furthermore, no approach could be found including the analysis of dendritic cells from lung-draining lymph nodes.

\section{Materials and Methods}

The sensitization and allergen challenge of mice were performed as described before [4]. All experiments were carried out using C57BL/6 mice (8-12 weeks old, Charles River, Sulzfeld, Germany). Mice ( $n=38$ ) were sensitized by intraperitoneal injection of $10 \mu \mathrm{g}$ ovalbumin (Grade VI) emulsified in $1.5 \mathrm{mg}$ aluminium hydroxide in a total volume of $150 \mu \mathrm{L}$ on days 1,14 , and 21 . Control mice $(n=42)$ were sham-sensitized with $1.5 \mathrm{mg}$ alum in PBS. OVA provocation (1\% OVA Grad V in PBS for $20 \mathrm{~min}$ ) was performed on days 28 and 29 on all mice. Lungs were obtained and cut into small fragments, digested with collagenase, and DNAse and enriched by gradient centrifugation and magnetic depletion of granulocytes, lymphocytes, and erythrocytes. Bronchial lymph nodes cells were isolated by passing the tissue through a metal mesh, directly followed by the magnetic depletion of granulocytes, lymphocytes, and erythrocytes. Remaining cells isolated from lung tissue and bronchial lymph nodes were resuspended in PBS and stained for 30 minutes with $\alpha$-CD11c, $\alpha$-MHCII, and $\alpha$-CD 8 (BD Biosciences). After washing, the stained cells were analysed and DCs (CD11c $^{\text {high }}$ and MHCII ${ }^{\text {high }}$ ) were sorted by using a Mo-FloSystem (Cytomation). The total RNA was isolated using the RNeasy Mini Kit (Qiagen, Hilden, Germany) according to the manufacturer's recommendations. Samples for microarray analysis were generated by applying an mRNA-specific double linear amplification protocol (Affymetrix). Briefly, double-stranded CDNA was generated in vitro in a reverse transcription using the T7dT23 primer $\left(5^{\prime}\right.$-GGCCAGTGAATTGTAATACGACTCACTATAGGGAGGCGG(T)23-3'; Metabion, Planegg, Germany) and SuperScript II reverse transcriptase (Invitrogen, Karlsruhe, Germany), followed by a second-strand cDNA synthesis involving DNA polymerase I (Invitrogen) and E. coli DNA ligase (Invitrogen). For the first amplification round the Promega P1300 RiboMax Kit for T7 amplification (Promega, Mannheim, Germany) was used to synthesize unlabeled cRNA from the purified cDNA. A second amplification round was performed starting with the amplified cRNA and reverse transcription by using random hexamer primers (Pharmacia, Freiburg, Germany) and SuperScript II reverse transcriptase for the first-strand synthesis. The second-strand synthesis, again using T7dT23 primers and additional RNase $\mathrm{H}$ treatment, was performed as mentioned above. For the final amplification round, the GeneChip expression 3'-Amplification Reagent Kit for labeling (Affymetrix, San Francisco, CA, USA), producing biotinylated cRNA, was used. The quantity and quality of biotinylated cRNA were checked photometrically. Samples were fragmented and hybridized to a Mouse Genome 4302.0 Array (Affymetrix). Washing and staining were performed as recommended by the manufacturer. Analysis of microarray data was performed using GeneSpring GX 10.0 software (Agilent Technologies). The robust multiarray analysis (RMA) algorithm was used for normalization.

\section{Results and Discussion}

The experiments and sorting procedures were performed with animal groups of 5 to 7 animals and repeated to finally reach the number of 42 control mice and 38 asthmatic-like mice for the cell isolation from lung tissue and the number of 15 control and 15 asthmatic-like mice for the cell isolation from lung-draining lymph nodes. The cells were deep-frozen and pooled for analysis in the gene arrays. The yield of dendritic cells from lung tissue was as follows. From 42 control mice in total $121.000 \mathrm{CD}^{+}$and $843.000 \mathrm{CD}^{-}$dendritic cells $\left(\mathrm{CD}_{11 c^{+}} / \mathrm{MHCII}^{+}\right)$were obtained from the tissue of whole lungs. From 38 asthmatic-like mice in total $118.000 \mathrm{CD}^{+}$ and $740.000 \mathrm{CD}^{-}$dendritic cells were obtained again from the lung tissue. The yield of dendritic cells from the lungdraining lymph nodes was as follows. From 15 control mice in total 168.000 dendritic cells $\left(\mathrm{CD}_{11 \mathrm{c}^{+}} / \mathrm{MHCII}^{+}\right)$were obtained and from 15 asthmatic-like mice 140.000 dendritic cells were 


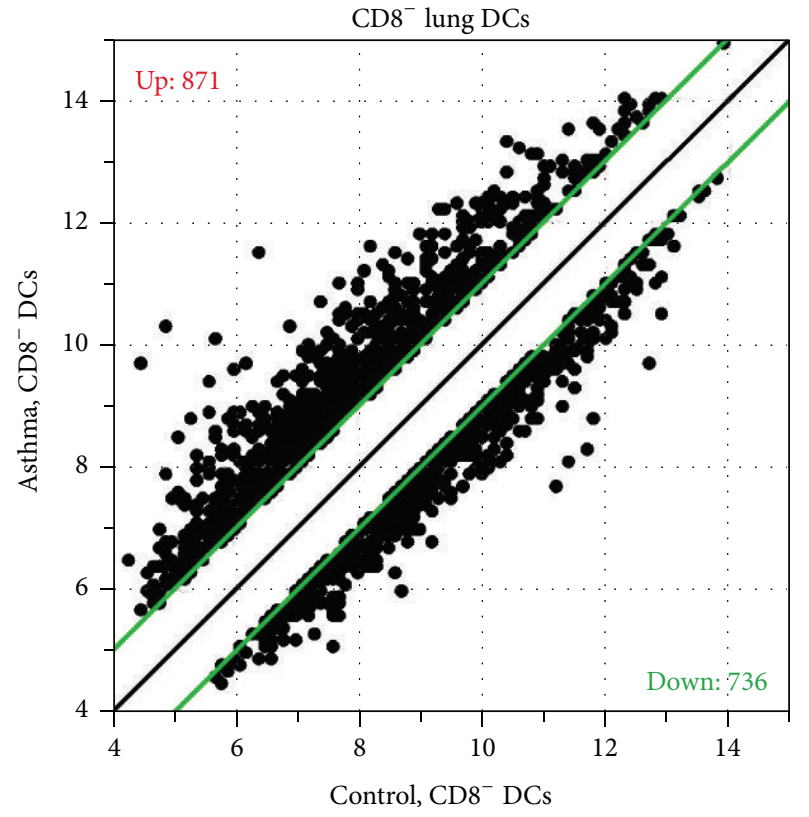

(a)

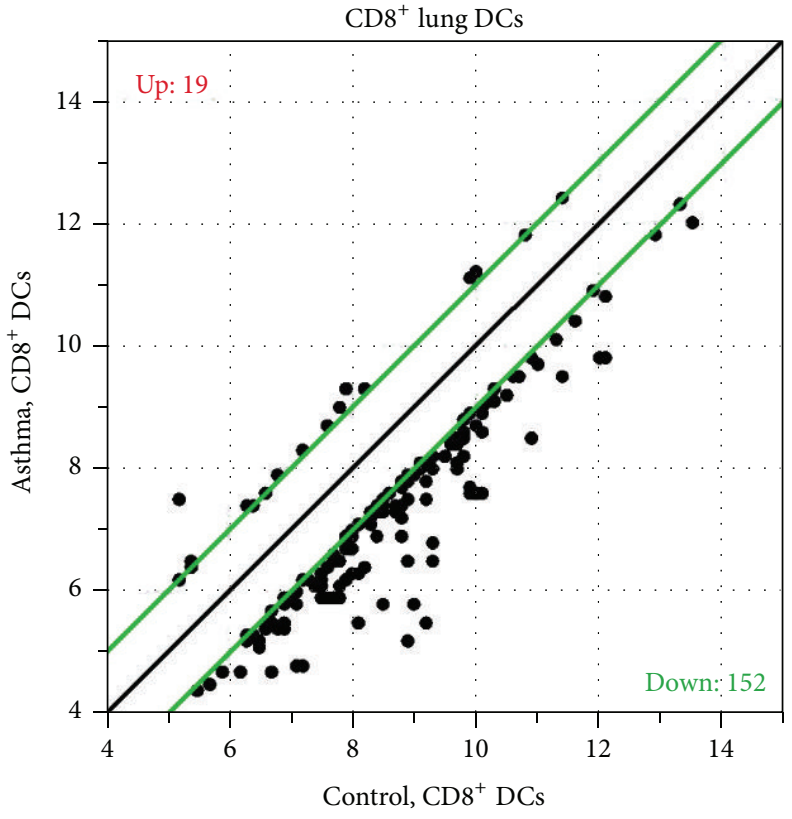

(b)

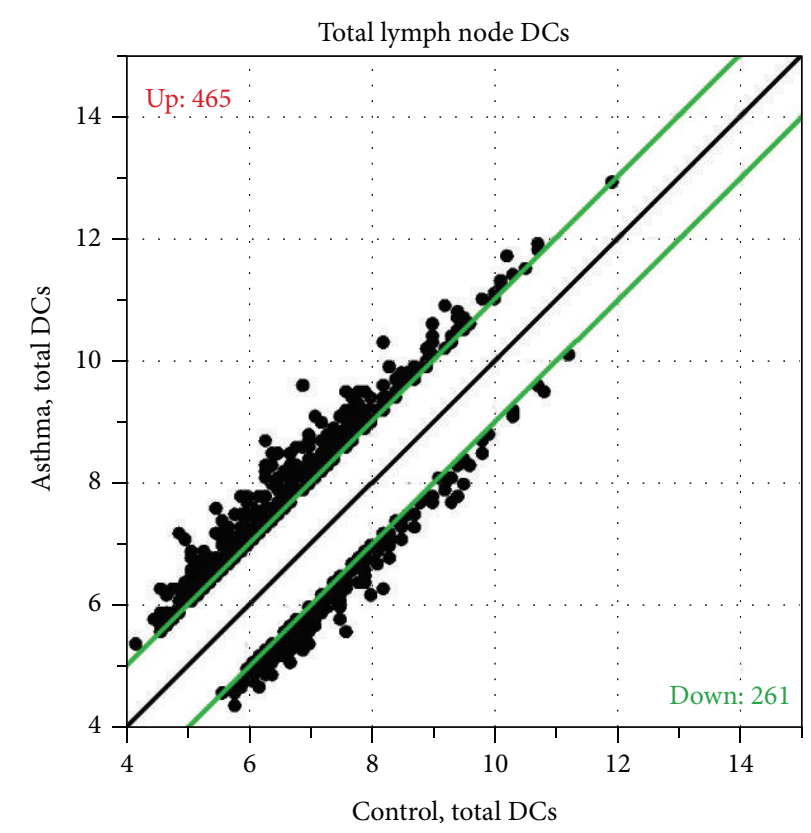

(c)

FIGURE 1: Scatterplots of DC microarrays. Normalized signal intensities of microarrays from the depicted DC subsets derived from lungs (a) and (b) and lung-draining lymph nodes (c) of asthma and control mice are plotted against each other. Only transcripts with a fold change of $> \pm 2$ (indicated by green lines) in the according comparison are shown. The numbers of up- and downregulated transcripts are depicted as well.

obtained. The reanalysis in flow cytometry led to a purity of at least $95 \%$, and in most experiments, the purity was 97 to 99\%.

In the $\mathrm{CD}^{-}$subset of lung tissue-derived dendritic cells 871 transcripts were upregulated with a fold change $> \pm 2$ in the comparison between induced allergic inflammation and controls (Figure 1). Again a high number, 736, of transcripts were downregulated. This is in contrast to the $\mathrm{CD}^{+}$subset in the same comparison, where only 19 transcripts were upregulated and 152 were downregulated (Figure 1). The $\mathrm{CD}^{+}$subpopulation is more constant and the few genes regulated are downregulated (Figure 1). This does not exclude a distinct function of the $\mathrm{CD} 8^{+} \mathrm{DC}$ subpopulation, like cross presentation of external antigen, for instance.

Since considerable lower numbers of dendritic cells could be obtained from the lung-draining lymph nodes, all 


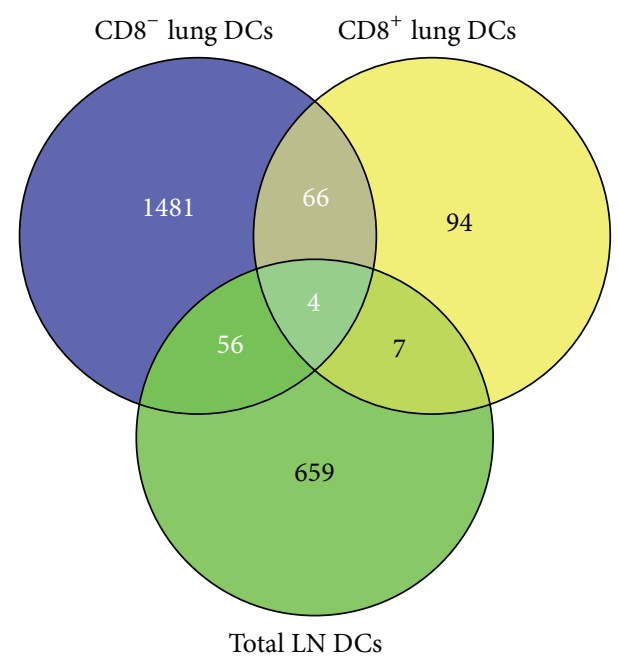

FIGURE 2: Venn diagram of regulated transcripts. The numbers and overlap of regulated transcripts ( $> \pm 2$-fold) in the depicted DC subset from lungs and lung-draining lymph nodes derived from asthma versus control animals are shown, respectively.

dendritic cells were analyzed and again those from asthmaticlike lungs were compared to those of control lungs. Here, 465 transcripts were upregulated and 261 were downregulated. One can speculate whether the $\mathrm{CD}^{-}$dendritic cells, being part of the sorted lymph node DCs were again those with the most regulated transcripts. It is known that, during inflammation, there is a constant flow of migrating dendritic cells to the draining lymph nodes, which induces massive changes in the cellular microenvironment in the lymph node [2]. It can be assumed that the majority of those migratory dendritic cells were $\mathrm{CD}^{-}$. But the transcriptional changes in the lymph nodes are moderate. This is interesting and may support a role of the lymph nodes in controlling the immune response and inflammation.

A Venn diagram (Figure 2) was used to display the overlap between regulated transcripts in all three populations: 66 were shared between the $\mathrm{CD} 8^{-}$and $\mathrm{CD}^{+}$dendritic cell population and 4 were shared by all dendritic cells, including the lymph node-derived ones. The small overlap of regulated genes amongst the three DC populations may underline their phenotypical distinctness. Moreover, the fact that the total lymph node DCs contain both $\mathrm{CD}^{+}$and $\mathrm{CD}^{-} \mathrm{DC}$ populations, but still show little overlap between the regulated genes found in asthmatic-lung-derived $\mathrm{CD}^{+}$or $\mathrm{CD}^{-} \mathrm{DCs}$ clearly shows that DC functions in asthma are dependent on the cellular environment. On the assumption that many $\mathrm{CD}^{-}$dendritic cells had migrated to the lymph nodes, the intense interaction with the stimulated micromilieus might have changed their phenotype.

In Tables 1 and 2, gene enrichment analysis of $\mathrm{CD}^{-}$ dendritic cells is presented because $\mathrm{CD}^{-}$dendritic cells seemed to be the most highly regulated during allergic inflammation. In the KEGG pathway enrichment significantly regulated genes have been found, which are involved in chemokine signaling, cytokine/receptor-interaction, Fc $\gamma$ Rmediated phagocytosis, and TLR signaling (Table 1). No significant KEGG pathway enrichment was found in the comparison of $\mathrm{CD}^{+}$dendritic cells (asthma versus control, see Supplemental Table 5 in Supplementary Materials available online at http://dx.doi.org/10.1155/2015/638032).

In the $\mathrm{GO}$ analysis, mainly changes of the plasma membrane and vesicles are prominent (Table 2). In the $\mathrm{CD} 8^{+}$ DC GO analysis, changes of the plasma membrane were prominent (Supplemental Table 5).

In the dendritic cells from draining lymph nodes consisting of total dendritic cells $\left(\mathrm{CD}^{-}\right.$and $\left.\mathrm{CD} 8^{+}\right)$, only few components are overrepresented (Supplemental Table 6). No significant changes occurred on cell surfaces but rather intracellular compartments (Table 3 ) seem to be modulated which might be related to the intense antigen processing and antigen presentation which is the core task of dendritic cells in the lymph node.

From the abundant regulated genes in the $\mathrm{CD} 8^{-}$dendritic cells highly upregulated genes were, amongst many others, distinct serpins, Arl5b, and Kif $3 b$ (supplemental material, excel sheet of regulated genes). Since it was not the primary aim of this study, no candidate genes were selected to perform confirming PCR analyses.

\section{Asthma-Induced Differential Gene Expression in $\mathrm{CD8}^{-}$Lung DCs}

The most prominent transcriptional alterations could be observed in the $\mathrm{CD}^{-}$DC compartment from asthmatic-like mice when compared to $\mathrm{CD} 8^{-} \mathrm{DC}$ from control mice. In general genes that are being upregulated more than 10 -fold under these conditions are, for example, Ear11, Abcd2, CD209e, Fabp1, and Slc7a2. Ear11, officially known as Rnase2a, encodes for the "Ribonuclease, RNase A family, 2A (liver, eosinophilderived neurotoxin)" protein. It is +34.7 -fold upregulated in asthmatic-like conditions. It has been previously reported to be asthma-induced in short-term, intermediate term, and long-term ovalbumin exposure of the lung [10]. $A b c d 2$ is upregulated +13.6 -fold. It encodes for the "ATP-binding cassette, subfamily D (ALD), member 2 ," a protein belonging to the large group of ATP-binding cassette $(\mathrm{ABC})$ transporters responsible for cross membrane transport of various substances. $A b c d 2$ is, for example, involved in the peroxisomal import of fatty acids. CD209e also known as Signr4 is a mouse homologue to the human DC-SIGN protein. DC-SIGN is a type II C-type lectin that functions as an adhesion molecule on the surface of dendritic cells. In the context of asthma, it has been reported that DC-SIGN on human MDDCs mediates cellular responses to, for example, Bermuda grass pollen antigens in vitro leading to the production of TNF- $\alpha$ [11]. Fabpl (fatty acid binding protein 1, liver) is upregulated +10.5 -fold in $\mathrm{CD}^{-}$DCs from asthmatic-like mice. That fatty acid binding proteins in principle are involved in DC function that has been demonstrated with regard to Fabp 4 (also known as $a P 2$ ), another protein from the same family with at least nine members [12]. Mice lacking $a P 2$ were shown to produce less IL-12 and TNF and were less potent in inducing T cell proliferation [13]. However, expression of Fabp1 on DCs in an asthmatic-like context has not been described so far. Slc7a2 stands for "solute carrier family 7 (cationic amino acid 
TABLE 1: KEGG pathway enrichment of 1607 regulated transcripts in CD8 ${ }^{-}$lung DCs.

\begin{tabular}{|c|c|c|c|c|c|}
\hline KEGG pathway & $\begin{array}{l}\text { KEGG } \\
\text { pathway } \\
\text { ID }\end{array}$ & $\begin{array}{c}\text { Total number of } \\
\text { genes in } \\
\text { pathway }\end{array}$ & $\begin{array}{l}\text { Number of } \\
\text { genes found }\end{array}$ & $\%$ genes found & $\begin{array}{c}\text { Bonferroni } \\
\text { corrected } \\
\text { term-enrichment } \\
P \text { value }\end{array}$ \\
\hline Lysosome & 4142 & 123 & 29 & 23.6 & $1.7 E-07$ \\
\hline Chemokine signaling pathway & 4062 & 185 & 29 & 15.7 & $1.7 E-03$ \\
\hline Peroxisome & 4146 & 79 & 17 & 21.5 & $1.8 E-03$ \\
\hline PPAR signaling pathway & 3320 & 81 & 17 & 21.0 & $2.5 E-03$ \\
\hline Cytokine-cytokine receptor interaction & 4060 & 248 & 34 & 13.7 & $5.7 E-03$ \\
\hline Fc gamma R-mediated phagocytosis & 4666 & 92 & 17 & 18.5 & $1.4 E-02$ \\
\hline Toll-like receptor signaling pathway & 4620 & 101 & 18 & 17.8 & $1.4 E-02$ \\
\hline Hematopoietic cell lineage & 4640 & 84 & 16 & 19.0 & $1.5 E-02$ \\
\hline B cell receptor signaling pathway & 4662 & 78 & 15 & 19.2 & $2.2 E-02$ \\
\hline Phagosome & 4145 & 177 & 25 & 14.1 & $3.6 E-02$ \\
\hline Focal adhesion & 4510 & 199 & 27 & 13.6 & $4.1 E-02$ \\
\hline
\end{tabular}

Transcripts that are found to be differentially regulated in $\mathrm{CD}^{-}$lung DCs from asthmatic-like versus healthy control mice were analyzed for statistical overrepresentation in KEGG pathway annotations using a one-sided hypergeometric test and Bonferroni $P$ value correction. Only KEGG pathways with a $P$ value $<0.05$ were considered to be overrepresented.

TABLE 2: Gene ontology analysis of 1607 regulated transcripts in CD8- lung DCs.

\begin{tabular}{|c|c|c|c|c|c|}
\hline Cellular component & GO ID & $\begin{array}{l}\text { Total number of } \\
\text { genes in category }\end{array}$ & $\begin{array}{l}\text { Number of genes } \\
\text { found }\end{array}$ & $\%$ genes found & $\begin{array}{c}\text { Bonferroni } \\
\text { corrected } \\
\text { term-enrichment } \\
P \text { value } \\
\end{array}$ \\
\hline Cytoplasm & 5737 & 2435 & 201 & 8.3 & $1.7 E-07$ \\
\hline External side of plasma membrane & 9897 & 145 & 29 & 20.0 & $2.4 E-07$ \\
\hline Cell surface & 9986 & 202 & 34 & 16.8 & $1.1 E-06$ \\
\hline Plasma membrane & 5886 & 1127 & 104 & 9.2 & $4.9 E-05$ \\
\hline Plasma membrane part & 44459 & 783 & 77 & 9.8 & $2.0 E-04$ \\
\hline Cytoplasmic part & 44444 & 1706 & 141 & 8.3 & $2.7 E-04$ \\
\hline Lytic vacuole & 323 & 57 & 11 & 19.3 & $2.8 E-02$ \\
\hline Lysosome & 5764 & 57 & 11 & 19.3 & $2.8 E-02$ \\
\hline Basolateral plasma membrane & 16323 & 79 & 13 & 16.5 & $4.4 E-02$ \\
\hline
\end{tabular}

Transcripts that are found to be differentially regulated in $\mathrm{CD}^{-}$lung DCs from asthmatic-like versus healthy control mice were analyzed for statistical overrepresentation in "cellular component" GO annotations using a one-sided hypergeometric test and Bonferroni $P$ value correction. Only GO terms with a $P$ value $<0.05$ were considered to be overrepresented.

transporter, y+ system), member 2" and belongs to the amino acid-polyamine-organocation family of transport proteins. $S l c 7 a 2$ is involved in the cellular uptake of arginine, lysine, and ornithine.

A comparably small group of genes in $\mathrm{CD}^{-}$DCs were found to be downregulated in an asthma-dependent manner. For example, the two heat shock proteins la and $1 \mathrm{~b}$ (Hspala and Hspalb) show a drastic loss of expression (-10.6- and 10.3-fold). Hspala and Hspalb are also known as heat-shock $70-\mathrm{kD}$ proteins $\mathrm{la}$ and $\mathrm{lb}$, respectively. They are involved in cellular stress responses and act as chaperons. Of note, the toll-like receptor 3 ( $T l r 3$ ) was also found to be downregulated -4.6 -fold. Tlr3 is a pattern recognition receptor sensing double-stranded RNA typically associated with viral infections.
As the cell surface is an important immunological interface of DCs, it is interesting to observe that genes whose protein products are associated with the cell surface are significantly enriched in the gene ontology analysis. In this category, genes like Htr2c, CD2, CD200r4, and CD22 may be of interest. The serotonin receptor $2 c(H t r 2 c)$ is highly upregulated (+7.1fold) under asthmatic-like conditions. Htr $2 c$ expression has been also demonstrated on epidermal DCs in context of a contact allergy model in mice [14]. The $\mathrm{T}$ lymphocyte surface antigen CD2 is +5.1-fold upregulated. Interestingly, $\mathrm{CD}^{+}$human $\mathrm{pDCs}$ were shown to comprise a distinct DC population producing higher amounts of IL-12p40 and expressing higher levels of costimulatory CD80 compared to $\mathrm{CD}^{-}$pDCs following an influenza A virus infection of the lung [15]. The CD200 receptor 4 (CD200r4) is upregulated 
TABLE 3: Gene ontology analysis of transcripts regulated in total DCs from lung-draining lymph nodes.

\begin{tabular}{|c|c|c|c|c|c|}
\hline Cellular component & GO ID & $\begin{array}{l}\text { Total number of } \\
\text { genes in category }\end{array}$ & $\begin{array}{l}\text { Number of } \\
\text { genes found }\end{array}$ & $\begin{array}{l}\text { \% genes } \\
\text { found }\end{array}$ & $\begin{array}{c}\text { Bonferroni corrected } \\
\text { term-enrichment } \\
P \text { value }\end{array}$ \\
\hline Intracellular & 5622 & 5869 & 239 & 4.1 & $5.0 E-11$ \\
\hline Intracellular part & 44424 & 5799 & 236 & 4.1 & $1.0 E-10$ \\
\hline Intracellular organelle & 43229 & 4906 & 205 & 4.2 & $2.2 E-09$ \\
\hline Intracellular membrane-bounded organelle & 43231 & 4389 & 174 & 4.0 & $2.4 E-05$ \\
\hline Intracellular organelle part & 44446 & 2247 & 103 & 4.6 & $4.3 E-05$ \\
\hline Cytoplasm & 5737 & 4078 & 162 & 4.0 & $8.2 E-05$ \\
\hline Nucleus & 5634 & 2346 & 104 & 4.4 & $1.8 E-04$ \\
\hline Cytoplasmic part & 44444 & 3065 & 127 & 4.1 & $3.2 E-04$ \\
\hline Intracellular non-membrane-bounded organelle & 43232 & 1127 & 58 & 5.1 & $8.6 E-04$ \\
\hline $\mathrm{Z}$ disc & 30018 & 54 & 8 & 14.8 & $1.2 E-02$ \\
\hline I band & 31674 & 61 & 8 & 13.1 & $2.8 E-02$ \\
\hline Cell cortex & 5938 & 99 & 10 & 10.1 & $4.6 E-02$ \\
\hline
\end{tabular}

Transcripts that are found to be differentially regulated in total lymph node DCs from asthmatic-like versus healthy control mice were analyzed for statistical overrepresentation in "cellular component" GO annotations using a one-sided hypergeometric test and Bonferroni $P$ value correction. Only GO terms with a $P$ value $<0.05$ were considered to be overrepresented.

+4.6 -fold on $\mathrm{CD}^{-}$DCs under allergic conditions and is a receptor for the Ox-2 ligand (also known as CD200). The CD200/CD200r-axis is thought to regulate myeloid cell activity. This is of particular interest since it has been recently reported in a rat asthma model that local delivery of recombinant CD200 strongly reduces OVA-induced lung accumulation of myeloid DCs in the lung [16]. CD22 also known as Siglec2 is upregulated 4 -fold in $\mathrm{CD}^{-}$DCs and belongs to the family of sialic-acid-binding lectins. Originally CD22 is thought to be a B cell restricted protein inhibiting the $\mathrm{B}$-cell antigen receptor (BCR) signaling [17]. However, there are reports demonstrating its expression also on pDCs [18].

On the other hand, the induction of asthma led in $\mathrm{CD}^{-}$ DCs also to the reduced expression of some surface proteins or at least their according transcripts. The killer cell lectinlike receptors $K l r b 1 b$ (-5.6-fold), $K l r d 1$ (-4.0-fold, also known as CD94) and Klrk1 (-2.1-fold, also known as $N k g 2 d)$ are all downregulated in $\mathrm{CD}^{-}$DCs compared to nonasthmatic-like conditions.

Interestingly, genes involved in antigen presentation like $\mathrm{H} 2-\mathrm{Oa}$ and $\mathrm{H} 2-\mathrm{Ob}$ which encodes for the histocompatibility 2 $\mathrm{O}$ region loci alpha and beta are slightly downregulated ( $\mathrm{H} 2-$ $\mathrm{Oa}$ : -2.7-fold, $\mathrm{H} 2-\mathrm{Ob}$ : -3.2-fold). Of note, the costimulatory protein CD86, working in conjunction with MHC class II proteins to activate $\mathrm{CD} 4^{+} \mathrm{T}$ cells, is downregulated as well (-2.2-fold).

With regard to the lysosomal compartment genes like Pla2g15, Sort1, and several cathepsins are regulated in $\mathrm{CD}^{-}$ DCs from asthmatic-like mice. Phospholipase A2 Group 15 (Pla2g15, +5.3-fold) is involved in the eicosanoid synthesis, a substance class also containing prostaglandins and leukotrienes which have pro- and anti-inflammatory potential.

Sortilin 1 (Sort1, +4.7-fold) very efficiently binds serum lipoproteins but can act as a multiligand type-1 receptor.
The group of cathepsins (cathepsins A, B, D, F, K, and L) is upregulated in $\mathrm{CD}^{-}$DCs from asthmatic-like lungs (Ctsa: +3.2-fold, Ctsb: +2.1-fold, Ctsd: +3.0-fold, Ctsf: +3.3-fold, Ctsk: +2.4 -fold, and Ctsl: +3.3 -fold). Cathepsins represent a group of endoproteases typically abundant in the lysosomal compartment and hydrolytically degrade, for example, the extracellular matrix and basal membranes.

In the functional group of "chemokine and cytokine signaling," genes like CD24 (+6.1-fold), Ccl24 (+6.1-fold), and $P d g f_{C}(+5.8$-fold) were highly upregulated. CD24 is upregulated +6.1 -fold. $\mathrm{CD} 24$ is a protein that is able to provide costimulatory signals to $\mathrm{T}$ cells and has been described to occur in the context of DC differentiation from $\mathrm{CD} 8 \alpha^{-}$to $\mathrm{CD} 8 \alpha^{+}$DCs [19]. Interestingly, it was reported that CD24deficient mice exhibit increased susceptibility to danger but not pathogen-associated molecular patterns [20]. Ccl24 is a chemokine also known as eotaxin-2 and is upregulated +6.1 fold in $\mathrm{CD}^{-}$DCs. $\mathrm{Ccl} 24$ is a ligand for Ccr3 and is able to recruit eosinophils [21]. Platelet-derived growth factor $\mathrm{C}$ polypeptide $(P d g f c,+5.8$-fold) is a potent mitogen for cells of mesenchymal origin [22]. Furthermore, genes like Lepr (leptin receptor; +4.7-fold), Adcy3 (adenylate cyclase 3; +3.7fold), Ccr1 (chemokine C-C motif receptor 1; +2.7-fold), Ccl6 (chemokine $\mathrm{C}-\mathrm{C}$ motif ligand $6 ;+2.4$-fold), Ccl8 (chemokine $\mathrm{C}-\mathrm{C}$ motif ligand 6; +2.4-fold), and Cxcr3 (chemokine C-X-C motif receptor $3 ;-4.5$-fold) were differentially regulated to a smaller extend.

\section{Conclusions}

Dendritic cells and their subsets play a key role in initiating and maintaining allergic inflammation. Dendritic cells are present in low numbers in lung tissue and in very low numbers in lung-draining lymph nodes. Gene analysis requires the separation of dendritic cells from huge numbers 
of animals, limiting the analysis of very rare subsets. The present analysis showed a regulation, up and down, of many more transcripts in the $\mathrm{CD} 8^{-}$conventional dendritic cells of the lung tissue as compared to the $\mathrm{CD} 8^{+}$DCs supporting the pathophysiological predominance of the $\mathrm{CD}^{-}$subset. Surprisingly, the transcriptional reaction in the dendritic cells of the draining lymph nodes was moderate indicating the role of the lymph nodes more as a stabilizer or controller than as a booster of the allergic inflammation. Conventional $\mathrm{CD} 8^{-}$and $\mathrm{CD}^{+}$dendritic cells are distinct subsets with differentiated roles in allergic inflammation. Further investigations will investigate whether the sorting of alternative dendritic cell subsets will show an overlap to those analyzed in this study.

\section{Conflict of Interests}

The authors declare that there is no conflict of interests regarding the publication of this paper.

\section{Authors' Contribution}

Thomas Tschernig, Christina Hartwig, and Andreas Jeron contributed equally to this study.

\section{Acknowledgments}

The authors thank Lothar Gröbe, HZI, for his excellent work on the sorting and reanalysis of dendritic cells. For her help with the manuscript and linguistic editing, we thank Ann Soether, Saarland University. The study was funded by the Deutsche Forschungsgemeinschaft (Sonderforschungsbereich 587).

\section{References}

[1] J. Deckers, F. Branco Madeira, and H. Hammad, "Innate immune cells in asthma," Trends in Immunology, vol. 34, no. 11, pp. 540-547, 2013.

[2] M. J. van Helden and B. N. Lambrecht, "Dendritic cells in asthma," Current Opinion in Immunology, vol. 25, no. 6, pp. 745754, 2013.

[3] M. Merad, P. Sathe, J. Helft, J. Miller, and A. Mortha, "The dendritic cell lineage: ontogeny and function of dendritic cells and their subsets in the steady state and the inflamed setting," Annual Review of Immunology, vol. 31, pp. 563-604, 2013.

[4] C. Hartwig, H. Constabel, D. Neumann, H. Gerd Hoymann, T. Tschernig, and G. M. N. Behrens, "Impact of boostering for the strength of asthma parameters and dendritic cell numbers in a C57BL/6 model of allergic airway inflammation," Experimental and Toxicologic Pathology, vol. 60, no. 6, pp. 425-434, 2008.

[5] B. T. Edelson, K. C. Wumesh, R. Juang et al., "Peripheral CD103 ${ }^{+}$ dendritic cells form a unified subset developmentally related to $\mathrm{CD} 8 \alpha^{+}$conventional dendritic cells," Journal of Experimental Medicine, vol. 207, no. 4, pp. 823-836, 2010.

[6] J. A. Villadangos and W. R. Heath, "Life cycle, migration and antigen presenting functions of spleen and lymph node dendritic cells: limitations of the Langerhans cells paradigm," Seminars in Immunology, vol. 17, no. 4, pp. 262-272, 2005.

[7] J. Y. Liu, P. Lu, L. Z. Hu et al., "CD8 $\alpha^{-}$DC is the major DC subset which mediates inhibition of allergic responses by Schistosoma infection," Parasite Immunology, vol. 36, no. 12, pp. 647-657, 2014.

[8] X. Gao, H. Bai, J. Cheng et al., "CD $8 \alpha^{+}$and $\mathrm{CD} 8 \alpha^{-}$DC subsets from BCG-infected mice inhibit allergic Th2-cell responses by enhancing Thl-cell and Treg-cell activity respectively," European Journal of Immunology, vol. 42, no. 1, pp. 165-175, 2012.

[9] E. L. Gautiar, T. Shay, J. Miller et al., "Gene-expression profiles and transcriptional regulatory pathways that underlie the identity and diversity of mouse tissue macrophages," Nature Immunology, vol. 13, no. 11, pp. 1118-1128, 2012.

[10] E. di Valentin, C. Crahay, N. Garbacki et al., "New asthma biomarkers: lessons from murine models of acute and chronic asthma," The American Journal of Physiology - Lung Cellular and Molecular Physiology, vol. 296, no. 2, pp. L185-L197, 2009.

[11] S.-C. Hsu, C.-H. Chen, S.-H. Tsai et al., "Functional interaction of common allergens and a C-type lectin receptor, dendritic cell-specific ICAM3-grabbing non-integrin (DC-SIGN), on human dendritic cells," Journal of Biological Chemistry, vol. 285, no. 11, pp. 7903-7910, 2010.

[12] M. Furuhashi and G. S. Hotamisligil, "Fatty acid-binding proteins: role in metabolic diseases and potential as drug targets," Nature Reviews Drug Discovery, vol. 7, no. 6, pp. 489-503, 2008.

[13] M. S. Rolph, T. R. Young, B. O. V. Shum et al., "Regulation of dendritic cell function and $\mathrm{T}$ cell priming by the fatty acidbinding protein AP2," Journal of Immunology, vol. 177, no. 11, pp. 7794-7801, 2006.

[14] H. El-nour, L. Lundeberg, A. Boman, D. Abramowski, M. Holst, and K. Nordlind, "The expression and functional significance of the serotonin ${ }_{2 C}$ receptor in murine contact allergy," Experimental Dermatology, vol. 16, no. 8, pp. 644-650, 2007.

[15] T. Matsui, J. E. Connolly, M. Michnevitz et al., "CD2 distinguishes two subsets of human plasmacytoid dendritic cells with distinct phenotype and functions," Journal of Immunology, vol. 182, no. 11, pp. 6815-6823, 2009.

[16] J.-F. Lauzon-Joset, A. Langlois, L. J. A. Lai et al., "Lung CD200R activation abrogates airway hyperresponsiveness in experimental asthma," American Journal of Respiratory Cell and Molecular Biology, 2015.

[17] T. Dorner, A. Shock, and K. G. C. Smith, "CD22 and autoimmune disease," International Reviews of Immunology, vol. 31, no. 5, pp. 363-378, 2012.

[18] E. Z. Reineks, E. S. Osei, A. Rosenberg, J. Auletta, and H. J. Meyerson, "CD22 expression on blastic plasmacytoid dendritic cell neoplasms and reactivity of anti-CD22 antibodies to peripheral blood dendritic cells," Cytometry Part B-Clinical Cytometry, vol. 76, no. 4, pp. 237-248, 2009.

[19] G. M. Del Hoyo, P. Martín, C. F. Arias, A. R. Marín, and C. Ardavín, "CD8alpha ${ }^{+}$dendritic cells originate from the CD8alpha ${ }^{-}$dendritic cell subset by a maturation process involving CD8alpha, DEC-205, and CD24 up-regulation," Blood, vol. 99, no. 3, pp. 999-1004, 2002.

[20] G.-Y. Chen, J. Tang, P. Zheng, and Y. Liu, "CD24 and siglec-10 selectively repress tissue damage-induced immune responses," Science, vol. 323, no. 5922, pp. 1722-1725, 2009.

[21] V. Provost, M.-C. Larose, A. Langlois, M. Rola-Pleszczynski, N. Flamand, and M. Laviolette, "CCL26/eotaxin-3 is more effective to induce the migration of eosinophils of asthmatics than CCL11/eotaxin-1 and CCL24/eotaxin-2," Journal of Leukocyte Biology, vol. 94, no. 2, pp. 213-222, 2013.

[22] D. G. Gilbertson, M. E. Duff, J. W. West et al., "Platelet-derived growth factor C (PDGF-C), a novel growth factor that binds to PDGF alpha and beta receptor," The Journal of Biological Chemistry, vol. 276, no. 29, pp. 27406-27414, 2001. 

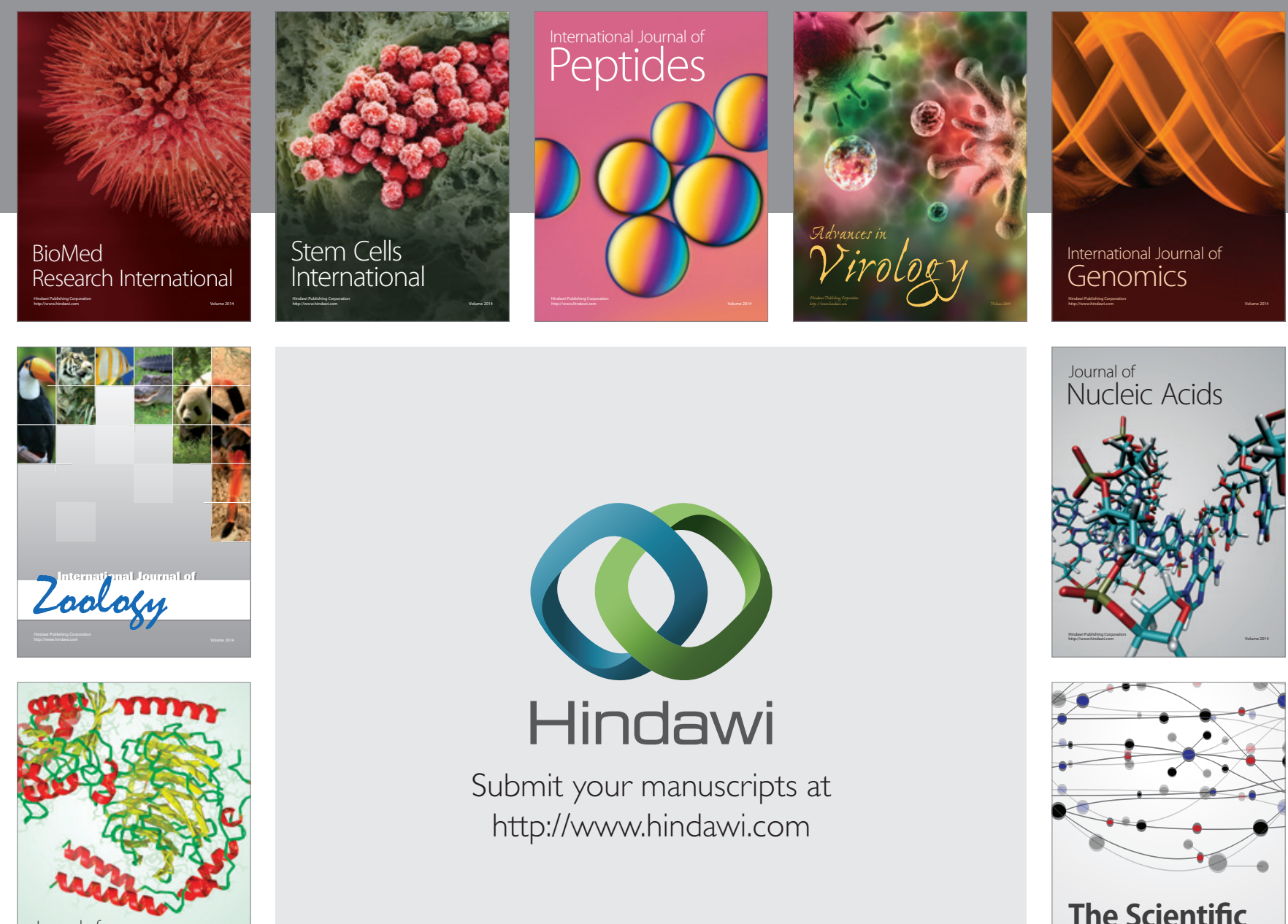

Submit your manuscripts at

http://www.hindawi.com

Journal of
Signal Transduction
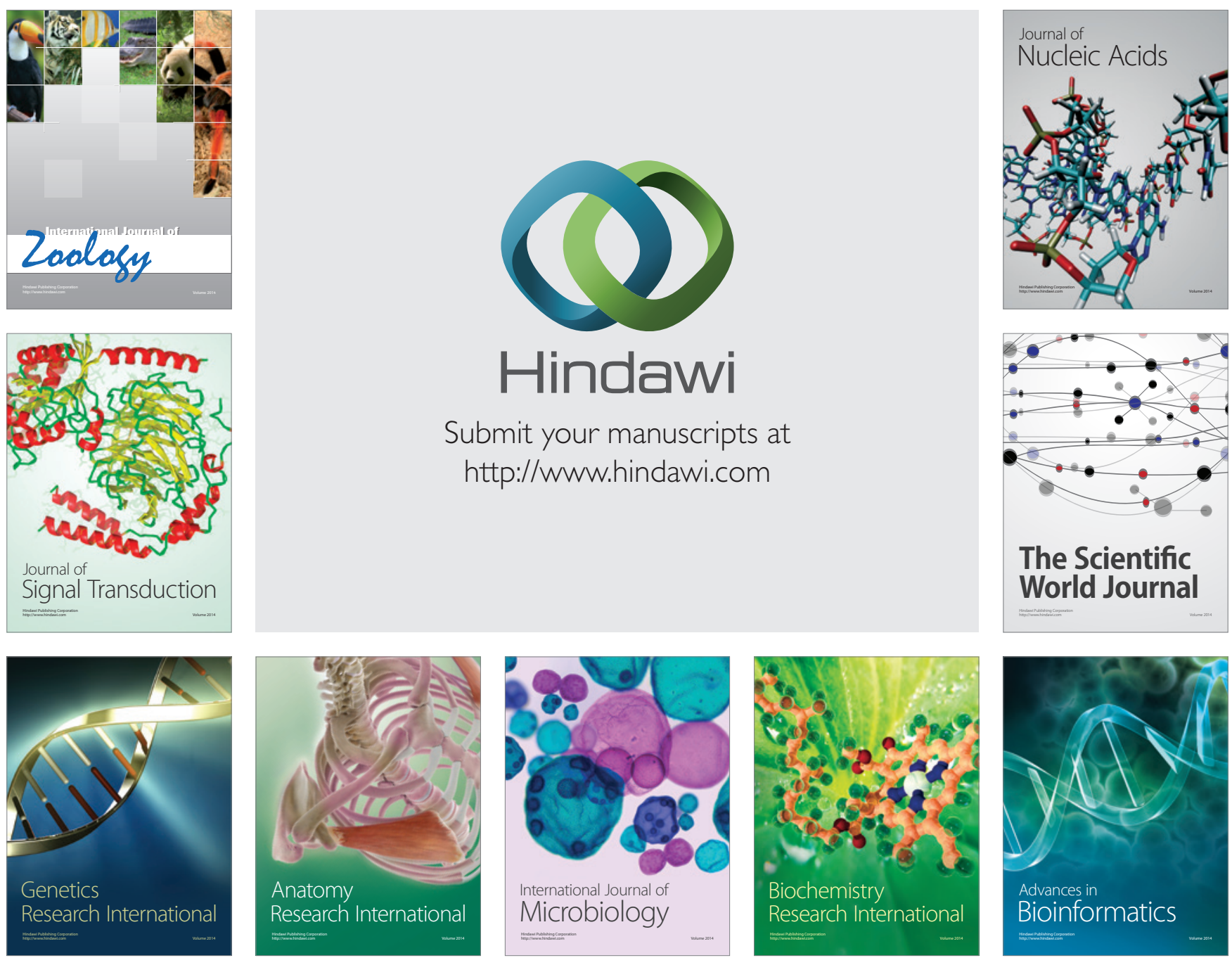

The Scientific World Journal
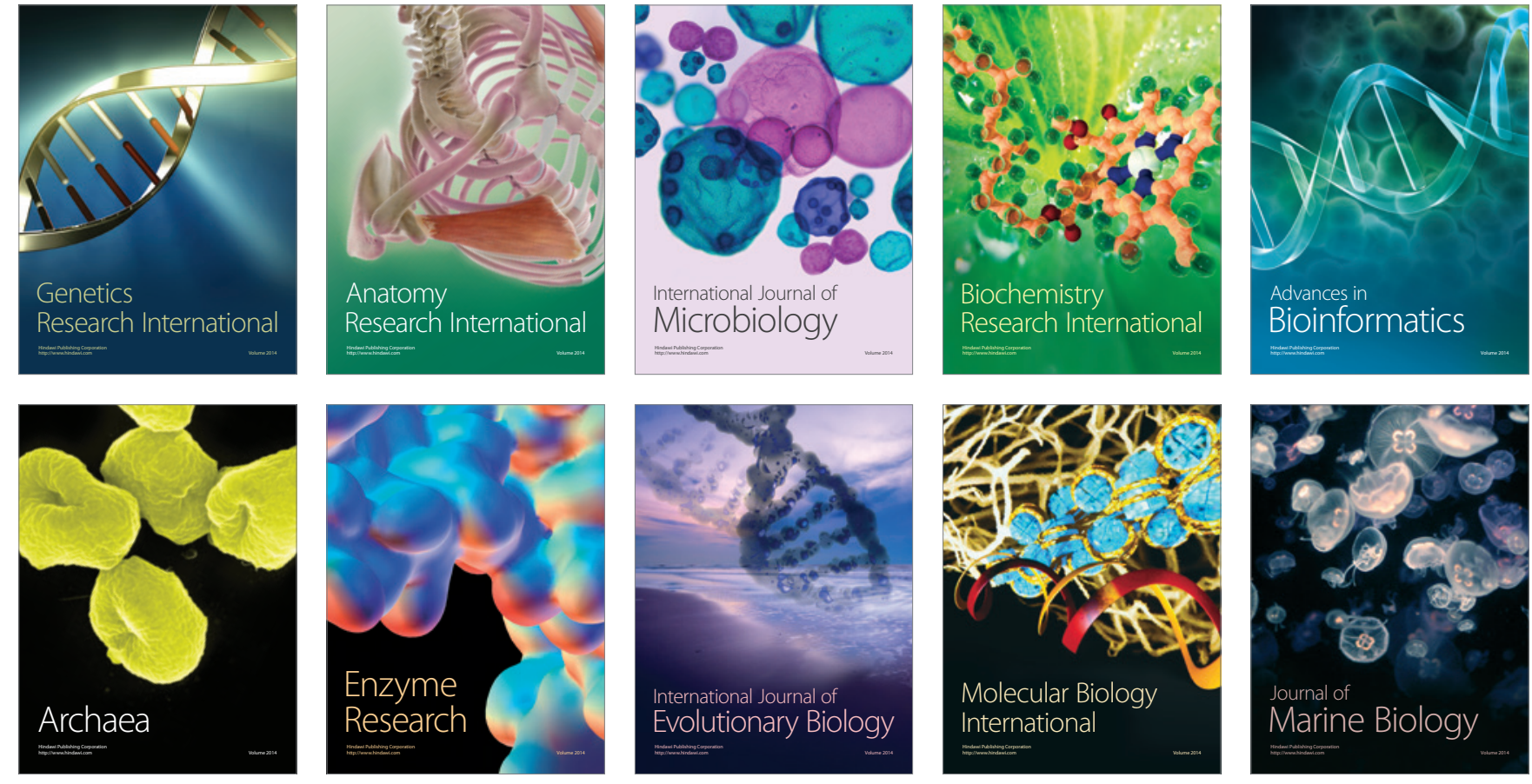